\title{
Molecular Gastronomy: Transforming Diets for Dysphagia
}

\author{
Rebecca Reilly, Farrell Frankel and Sari Edelstein* \\ Department of Nutrition, Simmons College, USA
}

Received: November 04, 2013; Accepted: November 14, 2013; Published: December 13, 2013

${ }^{*}$ Corresponding authors: Sari Edelstein, Department of Nutrition, Simmons College, S-334, Boston, MA 02115, USA, Tel: 617 521-2713; Fax: 617 521-

3002; E-mail: sari.edelstein@simmons.edu

\begin{abstract}
The purpose of our research is to utilize molecular gastronomy to improve food textures for dysphagia diets, using techniques such as spherification, gelification, and emulsification. It is important for individuals with dysphagia to continue to consume the necessary vitamins and minerals to ensure good health. Molecular gastronomy can be used to enhance pureed dietsas more palatable and aesthetically appealing. Medical nutrition therapy for dysphagia calls for foods and beverages to be thickened at prescribed textures and consistencies to prevent choking and aspiration. There is quite a range of unpalatable purees that are currently served to patients, inclusive of baby food and whole meals blenderized together and served in one large bowl. Molecular cuisine allows more variety for many with dysphagia, as food taste is one of few pleasures afforded these patients as a result of their condition and should replace undesirable one bowl meals still served in elder and healthcare facilities. The results of this research suggest that the use of molecular gastronomy in the preparation of pureed foods is favorable and rated highly. The implications of this research can be extended to nursing home facilities, hospitals and long-term care facilities, where patient's are often on pureed diets for dysphagia.
\end{abstract}

Keywords: Dysphagia; Dysphasia; Swallowing; Blenderized; Texturized; Medical nutrition therapy; Spherification; Gelification; Emulsification

\section{Review of Literature}

Dysphagia, is a word meaning disordered eating [1] and the medical term for swallowing difficulty as a result of either a mechanical obstruction or dysmotility [2]. Swallowing is a physiological process and entails the following steps: Food and liquid are transported from the oral cavity-mouth, into the stomach requiring up to 40 pairs of muscles and 8 nerves. The tongue and saliva work together to prepare food and liquid to move to the stomach. Saliva moistens food and triggers the swallowing reflex which alerts the larynx to close such that food doesn't travel down the trachea into the lungs. See Figure 1 for the swallowing process.

Dysphagia can present in a patient as a feeling of food/ drink sticking or a discomfort either in the throat. Occasionally, regurgitation, aspiration, or even hiccup may be the patient's presenting complaint [1]. Patients with degenerative diseases such as stroke, dementia, Parkinson's disease, Alzheimer's disease, heart disease, multiple sclerosis, and AIDS can present with dysphagia. In addition, the institutionalized elderly population has a high percentage rate of swallowing disorders and the consequence is can lead to undernourishment and dehydration [3].

\section{Diagnostic Considerations: Patient History}

Dysphagia is a common problem among the elderly and evaluation should start with careful history taking, to guide subsequent diagnostic testing and management. To enable the health practitioner to make an appropriate clinical evaluation, an in-depth, structured clinical history is important. Determining whether the issue is oropharyngeal or esophageal in origin is an important part of patient evaluation. The patient's symptoms will help determine the cause, whether it is oropharyngeal, esophageal or neuromuscular. Risk factors for esophageal and gastric cancers include smoking, alcohol use, and known family history of upper gastrointestinal (GI) malignancy [2].

Dysphagia that occurs only during swallowing of solids is more likely to indicate underlying mechanical obstruction, whereas when both solids and liquids are affected, dysmotility is the likely cause. Steps to find the origination of the swallowing difficulty are summarized below [2]:

Step 1: Define the presence of physical symptoms such as delayed or absent swallow initiation, cough post-swallowing, nasopharyngeal regurgitation, and repeated swallows to affect pharyngeal clearance, indicate potential oropharyngeal dysphagia.

Step 2: Define the location of symptoms as the throat is more likely to be oropharyngeal in origin and retrosternal region, which is more suggestive of an esophageal disorder.

Step 3: Define the duration and progression of symptoms is also important features as chronic stable symptoms suggest benign conditions such as peptic strictures.

\section{Diagnostic Considerations: Medical Procedures}

Accurate identification of the cause of dysphagia generally requires investigations that can be broadly divided into imaging and motility assessment. Gastroscopy is typically the first method chosen to exclude an obstructive lesion. Many techniques are 


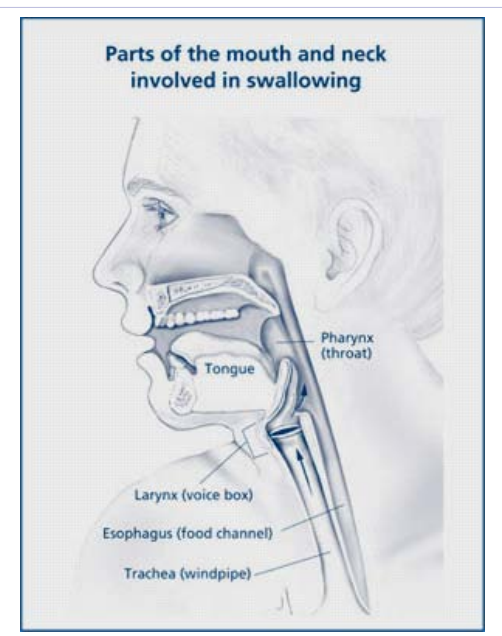

Figure 1: Swallowing is a complex process. Some 50 pairs of muscles and many nerves work to receive food into the mouth, prepare it, and move it from the mouth to the stomach. This happens in three stages.

1. During the first stage, called the oral phase, the tongue collects the food or liquid, making it ready for swallowing. The tongue and jaw move solid food around in the mouth so it can be chewed. Chewing makes solid food the right size and texture to swallow by mixing the food with saliva. Saliva softens and moistens the food to make swallowing easier. Normally, the only solid we swallow without chewing is in the form of a pill or caplet. Everything else that we swallow is in the form of a liquid, a puree, or a chewed solid.

2. The second stage begins when the tongue pushes the food or liquid to the back of the mouth. This triggers a swallowing response that passes the food through the pharynx, or throat. During this phase, called the pharyngeal phase, the larynx (voice box) closes tightly and breathing stops to prevent food or liquid from entering the airway and lungs.

3. The third stage begins when food or liquid enters the esophagus, the tube that carries food and liquid to the stomach. The passage through the esophagus, called the esophageal phase, usually occurs in about three seconds, depending on the texture or consistency of the food, but can take slightly longer in some cases, such as when swallowing a pill.

currently available for assessing esophageal motor function, although manometry and barium swallow remain the most clinically useful. High-resolution manometry with topography is now the new benchmark in assessing esophageal pressures and diagnosing conditions such as achalasia and esophageal spasm. Combining impedance with manometry in assessing bolus transit currently remains a research tool, as is the functional lumen imaging probe and high frequency intraluminal ultrasound. The choice of test depends on the clinical indication and the outcomes should be interpreted in conjunction with the patients' symptoms [2].

\section{Medical Nutrition Therapy}

Patients with dysphagia need to have diets that are appealing and modified in texture in order to get required calories, vitamins and other nutrients to ensure good health [3]. Another concern is serving improperly prepared beverages may contribute to medical complications such as dehydration if patients consume less fluid or aspiration of overly thin or thickened liquids, which may increase the risk of pneumonia [4]. Dietary modification to serve appetizing, yet proper consistency can be achieved by incorporating cooking and science.

For creating the proper consistency and texture for dyspahagia diets, we utilized molecular gastronomy. Molecular gastronomy was used to achieve food textures and tastes and the National Dysphagia Diet (NDD) was used as the guidelines for these textures [5]. Molecular gastronomy utilizes the food preparation techniques such as spherification, gelification, and emulsification. Spherification is the transforming of liquid into spheres. Usually a calcium bath can form soft spheres to appear, such as in tapioca pudding. Gelification consists of a liquid contained in a network. The network is usually of protein source (egg protein or meat gelatin). In foods, soy noodles and meringues are gels.

Emulsification is the dispersion of two liquids (one a liquid and one droplets) combined together with a stabilizer (i.e. soy lecithin or phospholipids) to prevent separation. In foods, gravies and salad dressings are commons emulsions [6].

The National Dysphagia Diet (NDD), now the national standard for dietary treatment of dysphagia, accounts for modifications in food textures, as well as liquids. Based on patient assessment, a customized dysphagia diet recommendation contains two plans: one for food texture and a second one for liquids. The following section explains the NDD levels for food textures and liquids [5].

\section{NDD Food Texture Levels}

\section{Level 1: Dysphagia Pureed Diet:}

i. This diet is designed for people who have moderate to severe dysphagia, with Poor oral phase abilities and reduced ability to protect their airway with complete supervision required.

ii. All foods will have a "soft mashed potato" or puddinglike consistency. Food should be of a mashed-potato consistency, not too runny or liquid. Pureed foods should be properly seasoned in the same way as regular foods. A blender or food processor is used to achieve proper consistency.

iii. Food may require additional liquids or thickening during preparation to achieve the proper consistency. Commerciallyprepared pureed foods are available as are formed purees, which provide a pureed food formed into the shape of the originating food: pureed chicken formed into a chicken breast or applesauce formed into an apple slice.

\section{Level 2: Dysphagia Mechanically Altered:}

i.This diet is a transition from the pureed textures to more solid textures. Some chewing ability is required.

ii.Foods are moist, soft-textured, and easily formed into a bolus. Examples include soft canned or cooked fruits, moistened ground meat, and well-cooked pasta.

iii.Aside from chopping or grinding meat, little special 
preparation is required. All foods must be well-cooked and hard fresh fruits and vegetables and other hard foods should be avoided.

\section{Level 3: Dysphagia Advanced:}

iii. This diet is a transition to a regular diet.

iv. Foods are prepared to nearly regular textures with the exception of very hard, sticky, or crunchy foods.

v. Foods should be moist. No special preparation is required.

vi. Avoid very dry foods, nuts, seeds, uncooked dry fruits, and tough meats.

\section{NDD Liquid Levels}

Thin liquids can be very difficult for people with dysphagia to swallow and are very easy to choke on or aspirate into the lungs. It may be recommended by the Speech and Swallow therapist or doctor to use thickened liquids. Over the counter products such as "thick-it" are available to thicken liquids to appropriate textures. Black, M. (2009) [7] Taste and texture are very personal issue to consider. Trained staff as well as the appearance and appeal affect the patient's willingness to accept the foods. The importance of keeping the patient hydrated and taking in enough calories and nutrients is critical in avoiding complications and improving outcomes from innumerable medical treatments [5].

The three recommended thickening levels for liquid diets are:

i. Nectar-Medium thickness- Thin liquids can be thickened with commercial thickeners or purchased pre-thickened to nectar-like thickness.

ii. Honey- Thicker than nectar. Room temperature honey consistency. Commercial thickeners can be used following instructions on the packages.

iii. Spoon Thick / Pudding-High viscous too thick for a straw. Commercial thickeners can be used following instructions on the packages.

iv. Commercial products are available to enhance food preparation for a puree or liquid diet and are among the following:

1. SimplyThick $®$ uses xanthan gum as thickener,water, xanthan gum, citric acid, and potassium sorbate (preservative)

Nectar, honey thicker. Spoon use 2 honey

Gelling for liquids (SimplyThick)

2. THICK \& EASY ${ }^{\circledR}$ products binds all of liquid in pureed foods with the food pulp, the risk of unbound liquid running down the throat is virtually eliminated.

THICK \& EASY $®$ food thickeners and Shape\&Serve ${ }^{\mathrm{TM}}$ are agglomerated, modified food starch that mixes easily with any type of pure food or THICK \& EASY® CLEAR for liquids without lumping.

For liquids, purees for molding and shaping slurry for cakes, bread. Can freeze the molded foods. (Thick \& Easy)

3. Resource and Puree Appeal-long list of ingredient.

Someone with food allergies could not use this-includes ingredients such as bread-

crumbs, dairy and egg plus corn syrup. Claims it gives the same textures as THICK \&

\section{EASY® (Resource and Puree Appeal)}

4. Thick It $®$ for beverages

Modified corn starch and maltodextrin (Thick It ${ }$ )

The challenge is how to implement the solution. Some residents/patients have trouble chewing so their food has to be blended. This can look fairly unappetizing on a plate. Special texturized blends of potatoes and vegetables are separate from the meat or fish. Putting these blends into apt molds, such as a fish one, or a cabbage one to make it look more appealing on the plate will be accepted more readily.

\section{Methods}

\section{Participants}

Participants were chosen using a convenience sample from the students taking Advanced Food Science at Simmons College. Participants were all female, with the exception of one male student. Participants were required to complete the dysphagia lab as part of their lab portion of the Advanced Food Science course. The sensory tasting was approved by the Simmons College Institutional Review Board.

\section{Assemble Equipment and Ingredients}

\section{Assemble Equipment:}

i. Blender or Food Processor

ii. Mixing Bowl

iii. Food Molds or Cookie Cutters

iv. Syringe

v. Tubing

vi. Pastry Bag

\section{Assemble ingredients:}

i. Texturized beef

ii. Sweet potatoes

iii. Marshmallows

iv. Yellow squash

v. Carrots

vi. Chicken

vii. Parsley

viii. Peas

ix. Tomatoes 


\author{
x. Noodles \\ xi. Basil \\ xii. Extra virgin olive oil \\ xiii. Peanut butter \\ xiv. Jelly \\ xv. Commercial Thickener \\ xvi. Food Coloring
}

\section{Pastry bag piping:}

iii. Insert coupler into the pastry bag and push it through to the smaller end

iv. Attach the pastry tip to the pastry bag, making sure there are no gaps between the coupler and bag. Special decorative tips may be used for aesthetics.

v. Kink the pastry bag so that mixture won't pour out the tip when being filled

vi. Fill the pastry bag and tighten

vii. Squeeze the pastry bag as mixture is pressed out from the tip onto the plate

\section{Food preparation}

\section{Gelification of Spaghetti:}

i. $\quad$ Fill a large bowl with ice water

ii. Use an immersion blender to puree to puree vegetables with $3 / 4$ c broth

iii. Combine puree with commercial starch thickener in a skillet and bring to a boil

iv. Place the mixture in a syringe

v. Attach silicon tubing to the end of syringe

vi. Fill the tubing with the mixture from the syringe

vii. Place syringe with tubing in a bowl of ice cold water for 3 minutes

viii. Fill the syringe with air and press to extract the spaghetti onto a plate. A pastry bag may be used as an alternative to a syringe and tube. Season to taste

\section{Spherification:}

Sweet Potatoes, yellow squash, carrots, peas, tomatoes

i. Pour canned vegetables and water into a blender

ii. Strain out liquid into a bowl, leaving cellulose, lignin or un-pureed substance in strainer

iii. Add commercial thickener until mixture reaches a pudding-like consistency

iv. Add food coloring as needed to match natural color of food v. Fill food molds or use pastry bag to present vegetable on plate. Season to taste

\section{Emulsification:}

Pesto sauce and parsley garnish

i. $\quad$ Puree $4 \mathrm{c}$ fresh basil in a blender

ii. Whisk together 1 egg yolk, 1/2t salt, 1/2t pepper

iii. Combine pureed basil with mixture in a bowl

iv. Slowly drizzle $1 / 2 \mathrm{c}$ extra virgin olive oil whisking to emulsify mixture

v. Place mixture in syringe and extract sauce onto plate

vi. Follow same process, substituting basil for parsley to make parsley garnish

\section{Marshmallow sauce:}

i. Melt marshmallows in a bowl

ii. Once marshmallows are melted, add water and thickening agent

iii. Place mixture in syringe and extract sauce onto plate

iv. As an alternative, place mixture in a pastry bag and extract onto plate

\section{Gravy Piping:}

i. $\quad$ Add gravy to syringe.

ii. Extract onto plate.

iii. As an alternative, use pastry bag with narrow tip and extract onto a plate

\section{Texturized Beef and Chicken:}

i. Puree meat in food processor or blender

ii. Add water until meat is a liquid mixture

iii. Add commercial thickener until mixture is a puddinglike consistency

iv. Fill meat shaped molds with mixture

v. Wait until mixture is firm in molds to plate the food

vi. An alternative to using molds is to pour mixture into a pastry bag and present on plate. Season to taste

\section{Peanut Butter and Jelly Sandwich:}

i. Assemble peanut butter and jelly sandwich on a plate

ii. In a separate bowl, mix commercial thickener with water until a pudding-like consistency

iii. Pour mixture over peanut butter and jelly sandwich and serve

\section{Results}

Sensory testing was performed on the products made in this research. The taste panel $(\mathrm{N}=60)$ found the food products to 
rank between 3-5 on a Likert Scale ( $1=$ not liked all at; $2=$ disliked somewhat; $3=$ =neutral; $4=$ liked somewhat; $5=$ =much liked). We were pleased with the results but also recommend more testing and tasting be done to achieve even higher scores. Additionally, taste panels consisted of college students who did not exhibit dysphagia symptoms. Figure 2 represents a pictorial of the food purees we made as described in the Methods section.

\section{Figure Guide}

i.Texturized beef with gravy piping and sweet potatoes with marshmallow garnish.

ii.Dental soft yellow squash with butterfly carrots.

iii.Pureed chicken mold with parsley garnish, peas and tomatoes.

iv.Texturized noodles with gravy, beef and pesto sauce.

v.Pureed peanut butter and jelly sandwich with side vegetables.

\section{Discussion}

The results of this study support the use of molecular gastronomy in the preparation of flavorful, aesthetically pleasing and sensory-appeal of foods for individuals with dysphasia. In order for patients with dysphasia to obtain the required calories, vitamins and minerals necessary for ensuring good health, their diets need to be modified. Molecular cuisine offers a means to control the texture and appeal of foods, so that food will be more appetizing to those with dysphasia. The results of this lab suggest that foods prepared using molecular gastronomy techniques, such as spherification, gelification and emulsification, have high sensory appeal, lending them to be more appetizing to those with dysphasia.

Following the Level 1 NDD guidelines for food and liquid texture, foods prepared using the gelification technique are the proper consistency for those individuals on a pureed food diet. The chicken and texturized beef prepared in this lab are pureed, yet they are formed in molds similar to the shape of the original food. The presentation of the pureed meats is appetizing while still adhering to the standardized texture guidelines for Level 1 NDD.
The molecular gastronomy technique of using a syringe and tubing to make pasta results in long strands of pasta with a texture meeting the Level 2 NDD guidelines. The pasta can be made using a variety of vegetables, so colors and taste may vary. Adding pesto sauce to the pasta completes the aesthetic appearance of the meal. The array of colors on each plate increases the appearance and appeal ratings of the meal. Adding food coloring to foods, especially canned vegetables, increases the appeal and can promote eating amongst individuals with dysphasia.

Overall, participants in the lab find that foods prepared using molecular cuisine are flavorful, visually appealing, and the appropriate texture for individuals with dysphasia. A limitation of the study is that temperature of the foods is not monitored. While preparing pureed chicken in a chicken mold is visually appealing, preparing the chicken puree at a temperature reflecting the normal temperature of a chicken breast brings the pureed food closer to resembling the actual food.

Further research can explore the cost benefit for molecular cuisine in comparison to purchasing supplements for individuals with dysphasia. Although the preparation for molecular cuisine is more cumbersome, the benefit to providing nutrient dense, appetizing and appealing food is potentially evident in an increase in the quality of life for individuals with dysphasia. Future research investigating the rating for quality of life in individuals with dysphasia who eat molecular cuisine as opposed to supplements or unpalatable purees found in many medical institutions is helpful in promoting the use of molecular cuisine $i$ $\mathrm{n}$ hospitals, nursing homes and other facilities.

\section{Conclusion}

The use of molecular gastronomy can serve as the new foundation of food preparation for dysphagia. Meals can be modernized to accomplish both the appropriate texture and taste for more sensory-appealing meals. The result of our project provided positive sensory evaluation $(\mathrm{N}=60)$ of flavorful, appealing food offerings that can encourage patients with dysphagia to eat more and enjoy meals which aids in their quality of life and nutritional well-being. This emerging cuisine can be used for improved medical nutrition therapy of dysphagia diets everywhere.

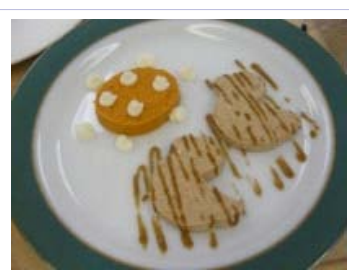

1

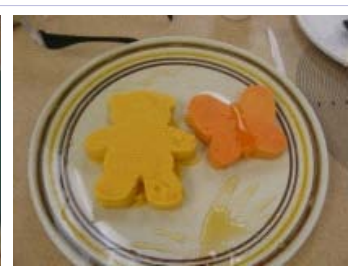

2

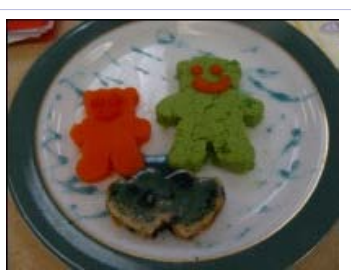

3

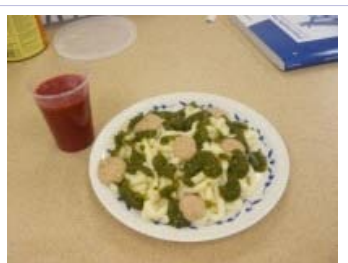

4

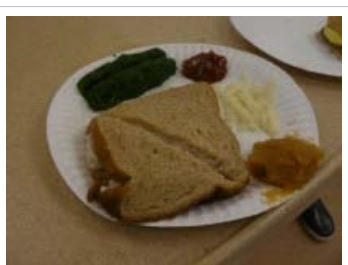

5

Figure 2: Appetizing pureed meals using food shapes.

1. Pureed beef with gravy piping and sweet potatoes with marshmallow garnish.

2. Pureed yellow squash with butterfly carrots.

3. Pureed chicken mold with parsley garnish, peas and tomatoes.

4. Pureed noodles with gravy, beef and pesto sauce.

5. Pureed peanut butter and jelly sandwich with side vegetables. 
Disadvantages to commercial supplements include acceptance and cost. Client acceptance may vary, as clients may perceive a flavor described as "medicinal" or tasting "like vitamin supplements." Some degree of protein and calorie malnutrition is strikingly prevalent in today's healthcare institutions [8].

Nutrition can reduce complications and improve outcomes for nearly every medical treatment imaginable - from surgery to cancer treatment to healing of fractures and more. It is possible for institutionalized elderly patients with dysphagia to eat better and increase body weight via a diversified, modified in texture and appealing oral diet that meets their nutrition needs [3].

Dietary variety has been associated with nutritional status in the elderly institutionalized population [4]. The general resident population enjoys and benefits from a wider selection of items every day on an à la carte menu. Elderly individuals with dysphagia might be expected to benefit from the same variety. Furthermore, the pleasant appearance and flavor of the modified texture foods (reformed or traditional) has been stated as important for sufficient and nutritionally balanced food intake. It is crucial that to find nutritive, varied, and appealing dietary solutions for elderly patients with dysphagia [3]. But whatever training is offered, it is undermined by the lack of any requirement for mandatory accredited training on nutrition or special diets for aides, kitchen staff or in home care. "If you can reduce the instances of malnutrition in the dysphagia community, you might reduce the numbers needing to go into care because malnutrition can cause confusion and frailty [9]." It is about "Treating older people with dignity [9]."

\section{References}

1. Paik, N. (2012). Dysphagia- overview. Medscape.

2. Kuo, P., Holloway, R.H., \& Nguyen, N.Q. (2012). Current and future techniques in the evaluation of dysphagia. Journal of Gastroenterology and Hepatology, 27(5), 873-881.

3. Germain, I., Dufresne, T., \& Gray-Donald, K. (2006). A novel dysphagia diet improves the nutrient intake of institutionalized elders. Journal of the American Dietetic Association, 106(10), 1614-1623.

4. Garcia, J.M., Chambers, E. 4th., Clark, M., Helverson, J., \& Matta, Z. (2010). Quality of care issues for dysphagia: modifications involving oral fluids. Journal of Clinical Nursing, 19(11-12), 1618-24.

5. Academy of Nutrition and Dietetics (2009). National dysphagia diet: standardization for optimal care.

6. Cazor, A., \& Lienard, C. (2012). Molecular cuisine. CRC Press, Taylor and Francis Group: New York.

7. Black, M. (2009). A multi level diet for those with a difficulty swallowing. Dysphagia Diet.

8. Understanding and implementing dysphagia diets. (2004). Association of Nutrition and Food Service Professionals.

9. Valios, N. (2007). Mean cuisine? Community Care, (1672), 34-36. 